\title{
Drivers of spatial and temporal variability of streamflow in the Incomati River basin
}

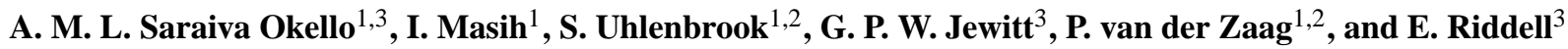 \\ ${ }^{1}$ UNESCO-IHE, Institute for Water Education, P.O. Box 3015, 2601 DA Delft, the Netherlands \\ ${ }^{2}$ Delft University of Technology, Department of Water Resources, P.O. Box 5048, 2600 GA Delft, the Netherlands \\ ${ }^{3}$ Centre for Water Resources Research, School of Agriculture, Earth and Environmental Science, \\ University of KwaZulu-Natal, Private Bag X01, Scottsville, 3209, South Africa
}

Correspondence to: A. M. L. Saraiva Okello (a.saraiva@unesco-ihe.org)

Received: 16 June 2014 - Published in Hydrol. Earth Syst. Sci. Discuss.: 29 July 2014

Revised: - - Accepted: 5 January 2015 - Published: 2 February 2015

\begin{abstract}
The Incomati is a semi-arid trans-boundary river basin in southern Africa, with a high variability of streamflow and competing water demands from irrigated agriculture, energy, forestry and industries. These sectors compete with environmental flows and basic human water needs, resulting in a "stressed" water resource system. The impacts of these demands, relative to the natural flow regime, appear significant. However, despite being a relatively well-gauged basin in South Africa, the natural flow regime and its spatial and temporal variability are poorly understood and remain poorly described, resulting in a limited knowledge base for water resource planning and management decisions. Thus, there is an opportunity to improve water management, if it can be underpinned by a better scientific understanding of the drivers of streamflow availability and variability in the catchment.

In this study, long-term rainfall and streamflow records were analysed. Statistical analysis, using annual anomalies, was conducted on 20 rainfall stations, for the period 19502011. The Spearman test was used to identify trends in the records on annual and monthly timescales. The variability of rainfall across the basin was confirmed to be high, both intraand inter-annually. The statistical analysis of rainfall data revealed no significant trend of increase or decrease. Observed flow data from 33 gauges were screened and analysed, using the Indicators of Hydrologic Alteration (IHA) approach. Temporal variability was high, with the coefficient of variation of annual flows in the range of 1 to 3.6. Significant declining trends in October flows, and low flow indicators, were also identified at most gauging stations of the Komati
\end{abstract}

and Crocodile sub-catchments; however, no trends were evident in the other parameters, including high flows. The trends were mapped using GIS and were compared with historical and current land use. These results suggest that land use and flow regulation are larger drivers of temporal changes in streamflow than climatic forces. Indeed, over the past 40 years, the areas under commercial forestry and irrigated agriculture have increased over 4 times.

\section{Introduction}

Global changes, such as climate change, population growth, urbanisation, industrial development and the expansion of agriculture, put huge pressure on natural resources, particularly water (Jewitt, 2006a; Milly et al., 2008; Vörösmarty et al., 2010; Miao et al., 2012; Montanari et al., 2013). In order to manage water in a sustainable manner, it is important to have a sound understanding of the processes that control its existence, the variability in time and space, and our ability to quantify that variability (Jewitt et al., 2004; Hu et al., 2011; Montanari et al., 2013; Hughes et al., 2014).

Water is critically important to the economies and social well-being of the predominantly rural populations of southern Africa, where environmental sustainability issues are increasingly coming into conflict with human development objectives, and where data are also scarce. The local economies and livelihoods of many southern African communities are strongly dependent on agriculture and fisheries, and water availability remains one of the main constraints to develop- 
ment in Africa (Jewitt, 2006a; Pollard and du Toit, 2009). Hydropower is also locally important, while a substantial amount of foreign income is derived from wildlife tourism in some countries of the region (Hughes et al., 2014).

Climate change intensifies the global hydrological cycle, leading to more frequent and variable extremes. For southern Africa, recent studies forecast an increase in the occurrence of drought due to decreased rainfall events (Shongwe et al., 2009; Rouault et al., 2010; Lennard et al., 2013). Furthermore, it is expected that temperatures will rise, and thus the hydrological processes driven by them will intensify (Kruger and Shongwe, 2004; Schulze, 2011). Compounding the effect of climate change are the increased pressures on land and water use, owing to increased population and the consequent requirements for food, fuel and fibre (Rockström et al., 2009; Warburton et al., 2010, 2012). Areas of irrigated agriculture and forestry have been expanding steadily over the past decades. Urbanisation also brings with it an increase in impervious areas and the increased abstraction of water for domestic, municipal and industrial purposes (Schulze, 2011).

In southern Africa, these pressures have led to changes in natural streamflow patterns. However, not many studies are available concerning the magnitude of such changes and what the main drivers are (Hughes et al., 2014). Projections on the impact of climate change on the water resources of South Africa were investigated by Schulze (2012) and streamflow trends of some southern Africa rivers have been analysed (Fanta et al., 2001; Love et al., 2010), but no such studies are available for the Incomati basin.

The Incomati is a semi-arid trans-boundary river basin in southern Africa, which is water-stressed because of high competing demands from, amongst others, irrigated agriculture, forestry, energy, environmental flow and basic human needs provision (DWAF, 2009b; TPTC, 2010). The impact of these demands, relative to the natural flow regime, is significant. Hence, there is an opportunity to improve water management, if a better scientific understanding of water resource availability and variability can be provided (Jewitt, 2006a).

The goal of this paper is to determine whether or not there have been significant changes in rainfall and streamflow during the time of record, and what the potential reasons for and implications of such changes are. The main research questions are the following.

- Does the analysis of precipitation and streamflow records reveal any persistent trends?

- What are the drivers of these trends?

- What are the implications of these trends for water management?

The variability and changes in rainfall and streamflow records were analysed and the possible drivers of changes were identified from the literature. The spatial variation of

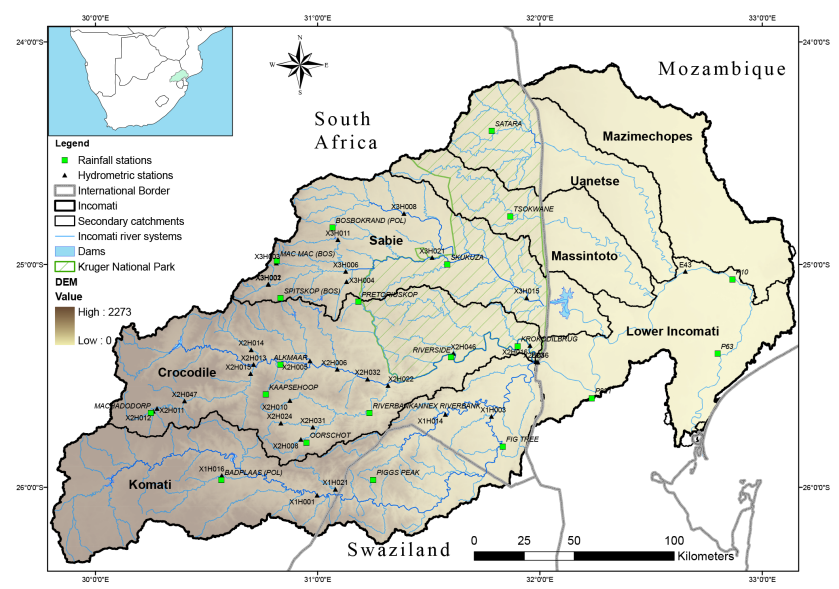

Figure 1. Map of the location of the study area, illustrating the main sub-catchments, the hydrometric and rainfall stations analysed, and the basin topography and dams.

trends in streamflow and their possible linkages with the main drivers are analysed. Based on the findings, approaches and alternatives for improved water resource management and planning are proposed.

\section{Methodology}

\subsection{Study area}

The Incomati River basin is located in the south-eastern part of Africa and it is shared by the Kingdom of Swaziland, the Republic of Mozambique and the Republic of South Africa (Fig. 1). The total basin area is approximately $46750 \mathrm{~km}^{2}$, of which $2560 \mathrm{~km}^{2}(5.5 \%)$ are in Swaziland, $15510 \mathrm{~km}^{2}(33.2 \%)$ in Mozambique and $28681 \mathrm{~km}^{2}$ $(61.3 \%)$ in South Africa. The Incomati watercourse includes the Komati, Crocodile, Sabie, Massintoto, Uanetse and Mazimechopes rivers and the estuary. The Komati, Crocodile and Sabie are the main sub-catchments, contributing about $94 \%$ of the natural discharge, with an area of $61 \%$ of the basin. The Incomati River rises in the Highveld and escarpment ( $2000 \mathrm{~m}$ above sea level) in the west of the basin and drops to the coastal plain in Mozambique. The general climate in the Incomati River basin varies from a warm to hot humid climate in Mozambique to a cooler dry climate in South Africa in the west. The mean annual precipitation of about $740 \mathrm{~mm} \mathrm{a}^{-1}$ falls entirely during the summer months (October to March). The Incomati (Fig. 1) can be topographically and climatically divided into three areas (TPTC, 2010):

- high escarpment, with a high rainfall (800 to 1600 $\mathrm{mma}^{-1}$ ), low temperatures (mean annual average of 10 to $16^{\circ} \mathrm{C}$ ) and lower potential evaporation (1600 to $2000 \mathrm{~mm} \mathrm{a}^{-1}$ ); 
Table 1. Summary of estimated natural streamflow, water demands in the Incomati basin in $10^{6} \mathrm{~m}^{3}$ per year (TPTC, 2010) and major dams $\left(>10^{6} \mathrm{~m}^{3}\right)$ (Van der Zaag and Vaz, 2003).

\begin{tabular}{|c|c|c|c|c|c|}
\hline & Natural MAR & First priority supplies & Irrigation supplies & Afforestration & Total water use \\
\hline Komati & 1332 & 141.5 & 621 & 117 & 879.5 \\
\hline Crocodile & 1124 & 74.7 & 482 & 158 & 714.7 \\
\hline Sabie & 668 & 30 & 98 & 90 & 218 \\
\hline Massintoto & 41 & 0.3 & 0 & 0 & 0.3 \\
\hline Uanetse & 33 & 0.3 & 0 & 0 & 0.3 \\
\hline Mazimechopes & 20 & 0 & 0 & 0 & 0 \\
\hline Lower Incomati & 258 & 1.5 & 412.8 & 0 & 414.3 \\
\hline Mozambique & 325 & & 412.8 & & \\
\hline South Africa & 2663 & & 961 & & \\
\hline Swaziland & 488 & & 240 & & \\
\hline Total & 3476 & 248 & 1614 & 365 & 2227 \\
\hline Tributary & Country & Major dam & Year commissioned & \multicolumn{2}{|c|}{ Storage capacity $\left(10^{6} \mathrm{~m}^{3}\right)$} \\
\hline Komati & South Africa & Nooitgedacht & 1962 & \multicolumn{2}{|c|}{81} \\
\hline Komati & South Africa & Vygeboom & 1971 & \multicolumn{2}{|c|}{84} \\
\hline Komati & Swaziland & Maguga & 2002 & \multicolumn{2}{|c|}{332} \\
\hline Komati & Swaziland & Sand River & 1966 & \multicolumn{2}{|c|}{49} \\
\hline Lomati & South Africa & Driekoppies & 1998 & \multicolumn{2}{|c|}{251} \\
\hline Crocodile & South Africa & Kwena & 1984 & \multicolumn{2}{|c|}{155} \\
\hline Crocodile & South Africa & Witklip & 1979 & \multicolumn{2}{|c|}{12} \\
\hline Crocodile & South Africa & Klipkopje & 1979 & \multicolumn{2}{|c|}{12} \\
\hline Sabie & South Africa & Da Gama & 1979 & \multicolumn{2}{|c|}{14} \\
\hline Sabie & South Africa & Injaka & 2001 & \multicolumn{2}{|c|}{120} \\
\hline Sabie & Mozambique & Corumana & 1988 & \multicolumn{2}{|c|}{879} \\
\hline Total & & & & \multicolumn{2}{|c|}{1989} \\
\hline
\end{tabular}

* First priority supplies include domestic and industrial uses.

- Highveld and middle Lowveld, which lie between the Drakensberg and the Lebombo Mountains, warmer than the escarpment (mean annual average of 14 to $22^{\circ} \mathrm{C}$ ), with rainfall that decreases towards the east (400 to $800 \mathrm{~mm} \mathrm{a}^{-1}$ ) and high potential evaporation (2000 to $\left.2200 \mathrm{~mm} \mathrm{a}^{-1}\right)$;

- coastal plain, located mostly in Mozambique, with higher temperatures (mean annual average of 20 to $26^{\circ} \mathrm{C}$ ) and lower rainfall (400 to $800 \mathrm{~mm} \mathrm{a}^{-1}$ ) in the west, increasing eastward towards the coast, where there is also high potential evaporation (2200 to $2400 \mathrm{~mm} \mathrm{a}^{-1}$ ).

The geology is complex, characterised by sedimentary, volcanic, granitic and dolomitic rocks, as well as Quaternary and recent deposits (Van der Zaag and Vaz, 2003). The soils in the basin are highly variable, ranging from moderately deep clayey loam in the west, to moderately deep sandy loam in the central areas and moderately deep clayey soils in the east. The dominant land uses in the catchment are commercial forest plantations of exotic trees (pine, eucalyptus) in the escarpment region, dryland crops (maize) and grazing in the Highveld region, and irrigated agriculture (sugarcane, vegetables and citrus) in the Lowveld (DWAF, 2009b; Riddell et al., 2013). In the Mozambican coastal plains, sugarcane and subsistence farming dominate. A substantial part of the basin has been declared a conservation area, which includes the recently established Greater Limpopo Transfrontier Park (the Kruger National Park in South Africa and the Limpopo National Park in Mozambique are part of it) (TPTC, 2010).

The level of water abstraction in the Incomati River is very high, and the actual water demand is projected to increase in the future, as a result of further economic development and population growth (Nkomo and van der Zaag, 2004; LeMarie et al., 2006; Pollard et al., 2011). The consumptive use of surface water amounts to more than 1880 million cubic metres per annum $\left(10^{6} \mathrm{~m}^{3} \mathrm{a}^{-1}\right)$, which represents $51 \%$ of the average amount of surface water generated in the basin (Van der Zaag and Vaz, 2003). The major water consumers (see Tables 1 and 2), accounting for $91 \%$ of all consumptive water use, are the irrigation and forestry sectors, followed by inter-basin water transfers to the Umbeluzi basin and the Olifants catchment in the Limpopo basin (Van der Zaag and Vaz, 2003; DWAF, 2009b; TPTC, 2010). Since the 1950s, the area of irrigated agriculture and forestry has increased steadily, particularly in the Komati and Crocodile systems (Table 2). 
Table 2. Land-use and water-use change from the 1950s to 2004 in the Komati, Crocodile and Sabie sub-catchments. Source: adapted from TPTC (2010).

\begin{tabular}{llrrrr}
\hline & & $1950 \mathrm{~s}$ & $1970 \mathrm{~s}$ & 1996 & 2004 \\
\hline \multirow{6}{*}{ Komati } & Irrigation area $\left(\mathrm{km}^{2}\right)$ & 17.6 & 144.1 & 385.1 & 512.4 \\
& Afforested area $\left(\mathrm{km}^{2}\right)$ & 247 & 377 & 661 & 801 \\
& Domestic water use $\left(10^{6} \mathrm{~m}^{3} \mathrm{a}^{-1}\right)$ & 0.5 & 7.7 & 15.5 & 19.7 \\
& Industrial and mining water use $\left(10^{6} \mathrm{~m}^{3} \mathrm{a}^{-1}\right)$ & 0 & 0 & 0.5 & 0.5 \\
& Water transfers out $\left(10^{6} \mathrm{~m}^{3} \mathrm{a}^{-1}\right):$ & & & & \\
& To power stations in South Africa & 3.4 & 103 & 98.1 & 104.7 \\
& To irrigation in Swaziland outside Komati & 0 & 111.8 & 122.2 & 121.8 \\
\hline \multirow{5}{*}{ Crocodile } & Afforested area $\left(\mathrm{km}^{2}\right)$ & 92.8 & 365.8 & 427 & 510.7 \\
& Irrigation area $\left(\mathrm{km}^{2}\right)$ & 375 & 1550 & 1811 & 1941 \\
& Domestic water use $\left(10^{6} \mathrm{~m}^{3} \mathrm{a}^{-1}\right)$ & 3 & 12.2 & 33.6 & 52.4 \\
& Industrial and mining water use $\left(10^{6} \mathrm{~m}^{3} \mathrm{a}^{-1}\right)$ & 0.1 & 7.5 & 19.8 & 22.3 \\
\hline \multirow{3}{*}{ Sabie } & 27.7 & 68.4 & 113.4 & 127.6 \\
& Irrigation area $\left(\mathrm{km}^{2}\right)$ & 428 & 729 & 708 & 853 \\
& Afforested area $\left(\mathrm{km}^{2}\right)$ & 2.4 & 5.3 & 13 & 26.7 \\
& Domestic water use $\left(10^{6} \mathrm{~m}^{3} \mathrm{a}^{-1}\right)$ & 0 & 0 & 0 & 0 \\
\hline & Industrial and mining water use $\left(10^{6} \mathrm{~m}^{3} \mathrm{a}^{-1}\right)$ & 0 & &
\end{tabular}

\subsection{Data and analysis}

\subsubsection{Rainfall}

Annual, monthly and daily rainfall data for southern Africa for the period of 1905 to 2000 were extracted from the Lynch (2003) database. The database consists of daily precipitation records for over 12000 stations in southern Africa, and data quality was checked and some data were patched. The main custodians of rainfall data are SAWS (South African Weather Service), SASRI (South Africa Sugarcane Research Institute) and ISCW (Institute for Soil Climate and Water). About 20 stations out of 374 available for Incomati were selected for detailed analysis. The selection criteria were the quality of data, evaluated by the percentage of reliable data in the database, and the representative spatial coverage of the basin. Eight of the 20 stations' time series were extended up to 2012, using new data collected from the SAWS.

The spatial and temporal heterogeneity in rainfall across the study area was characterised using statistical analysis and annual anomalies. The time series of annual and monthly rainfall from each station were subjected to the Spearman test in order to identify trends for the periods of 1950-2000 and 1950-2011. Two intersecting periods were chosen to evaluate the consistency of the trends. Due to natural climatic variability, there are sequences of wetter and drier periods, so some trends appearing in a specific period might be absent when a longer or shorter period is considered. The Pettitt test (Pettitt, 1979) is used to detect abrupt changes in the time series. Potential change points divide the time series into two sub-series. Then, the significance of the change in the mean and variance of the two sub-series is evaluated by $F$ and $t$ tests. Potential change points were evaluated with a 0.8 probability threshold and significance of change was assessed with $F$ and $t$ tests at the $95 \%$ confidence level (Zhang et al., 2008; Love et al., 2010). The annual and monthly time series were also analysed for the presence of serial correlation. Tests were carried out using SPELL-stat v.1.5.1.0B (Guzman and Chu, 2003).

\subsubsection{Streamflow}

Streamflow data of 104 gauging stations in South Africa (obtained from the Department of Water Affairs - DWA) and two gauging stations in Mozambique (obtained from ARASul) were used in this study. A long time series of flow data was not available in Swaziland. Based on the quality of the data, time series length, influence of infrastructure (dams, canals) and spatial distribution, 33 stations were selected for detailed analysis (Table 3 and Fig. 2). As this catchment is highly modified, very few stations could be considered not impacted by human interventions. Data from pristine catchments can reveal the dynamics of natural variability of streamflow and isolate the impacts of climate change on streamflow. An analysis of the indicators of hydrologic alteration was conducted to identify patterns and trends of the streamflow record (a single period analysis for the entire time series and for the period of 1970-2011), as well as to assess the impact of infrastructure on the streamflow (two-period analysis, before and after the major infrastructure development). 


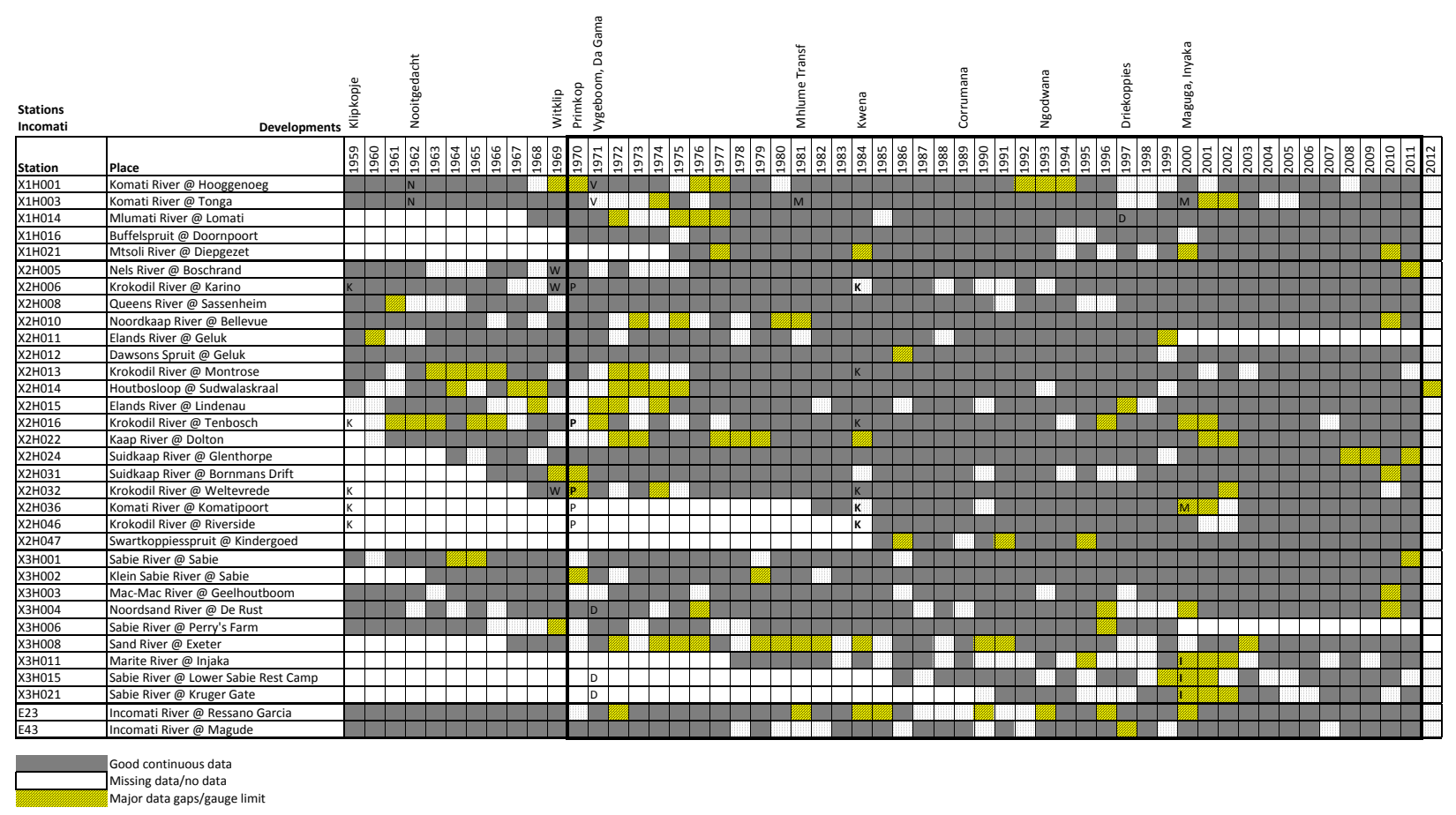

Figure 2. Streamflow data used on this study, with indication of time series length, data quality, and missing data. Major developments in the basin, such as dams, are on the top horizontal line in the year they were commissioned; indication is made of the gauges affected by the developments by the initial letter of the dam.

\subsubsection{Indicators of hydrologic alteration}

The US Nature Conservancy developed a statistical software program known as the Indicators of Hydrologic Alteration (IHA) for assessing the degree to which human activities have changed flow regimes. The IHA method (Richter et al., 1996, 2003; Richter and Thomas, 2007) is based upon the concept that hydrologic regimes can be characterised by five ecologically relevant attributes, listed in Table 4: (1) magnitude of monthly flow conditions; (2) magnitude and duration of extreme flow events (e.g. high and low flows); (3) the timing of extreme flow events; (4) frequency and duration of high low flow pulses; and (5) the rate and frequency of changes in flows. It consists of 67 parameters, which are subdivided into two groups - 33 IHA parameters and 34 environmental flow component parameters. These hydrologic parameters were developed based on their ecological relevance and their ability to reflect human-induced changes in flow regimes across a broad range of influences including dam operations, water diversions, groundwater pumping, and landscape modification (Mathews and Richter, 2007). 33 selected gauges from the Incomati basin were analysed with this method using daily flow data. Many studies successfully applied the methodology of Indicators of Hydrologic Alteration, in order to assess impacts on streamflow caused by anthropogenic drivers (Maingi and Marsh, 2002; Taylor et al., 2003; Mathews and Richter, 2007; De Winnaar and Jewitt,
2010; Masih et al., 2011). In the case of the present study, the indicators of the magnitude of monthly flow, the magnitude and duration of extreme flow, as well as timing, were analysed for the period 1970-2011, to assess whether consistent trends of increase or decrease of the hydrological indicators were present.

The IHA software was used to identify trends of the streamflow time series, based on the regression of least squares. This trend is evaluated with the $P$ value, and only trends with $P \leq 0.05$ were considered significant trends, the value of the slope of the trend line indicating an increasing or decreasing trend. This information was compiled and mapped for the various hydrological indicators using $\mathrm{Ar}$ cGIS 9.3.

\subsubsection{Land-use analysis}

Land use was analysed based on secondary data because most of the current forestry plantations were already established on the 1970s, when remote sensing maps became available. Additionally, a map of current land use (2011) (Jarmain et al., 2013) and the land use of 2000 (Van den Berg et al., 2008) were compared with the maps of trends of indicators of hydrologic alteration. Where the occurrence of trends in a flow regime was consistent with the changes in land use, this the temporal evolution of the land-use changes was further investigated. 
Table 3. Hydrometric stations analysed, location, catchment area, data length and missing data.

\begin{tabular}{|c|c|c|c|c|c|c|c|c|}
\hline & Station & Latitude & Longitude & River and location & $\begin{array}{l}\text { Catchment } \\
\text { area }\left(\mathrm{km}^{2}\right)\end{array}$ & $\begin{array}{r}\text { Data } \\
\text { available }\end{array}$ & $\begin{array}{r}\text { Period analysed } \\
\text { for trends }\end{array}$ & $\begin{array}{r}\text { Missing } \\
\text { data }\end{array}$ \\
\hline \multirow{5}{*}{ Komati } & X1H001 & -26.04 & 31.00 & Komati River, Hooggenoeg & 5499 & 1909-2012 & 1970-2011 (42 years) & $8.0 \%$ \\
\hline & $\mathrm{X} 1 \mathrm{H} 003$ & -25.68 & 31.78 & Komati River, Tonga & 8614 & 1939-2012 & 1970-2011 (42 years) & $6.8 \%$ \\
\hline & X1H014 & -25.67 & 31.58 & Mlumati River, Lomati & 1119 & $1968-2012$ & 1978-2011 (34 years) & $0.5 \%$ \\
\hline & X1H016 & -25.95 & 30.57 & Buffelspruit, Doornpoort & 581 & 1970-2012 & 1970-2011 (42 years) & $3.4 \%$ \\
\hline & $\mathrm{X} 1 \mathrm{H} 021$ & -26.01 & 31.08 & Mtsoli River, Diepgezet & 295 & $1975-2012$ & 1976-2011 (36 years) & $2.7 \%$ \\
\hline \multirow{17}{*}{ Crocodile } & $\mathrm{X} 2 \mathrm{H} 005$ & -25.43 & 30.97 & Nels River, Boschrand & 642 & 1929-2012 & 1970-2011 (42 years) & $0.8 \%$ \\
\hline & $\mathrm{X} 2 \mathrm{H} 006$ & -25.47 & 31.09 & Krokodil River, Karino & 5097 & 1929-2012 & 1970-2011 (42 years) & $0.1 \%$ \\
\hline & $\mathrm{X} 2 \mathrm{H} 008$ & -25.79 & 30.92 & Queens River, Sassenheim & 180 & 1948-2012 & 1970-2011 (42 years) & $0.5 \%$ \\
\hline & $\mathrm{X} 2 \mathrm{H} 010$ & -25.61 & 30.87 & Noordkaap River, Bellevue & 126 & $1948-2012$ & 1970-2011 (42 years) & $5.7 \%$ \\
\hline & $\mathrm{X} 2 \mathrm{H} 011$ & -25.65 & 30.28 & Elands River, Geluk & 402 & 1956-1999 & 1957-1999 (43 years) & $0.9 \%$ \\
\hline & $\mathrm{X} 2 \mathrm{H} 012$ & -25.66 & 30.26 & Dawsons Spruit, Geluk & 91 & 1956-2012 & 1970-2011 (42 years) & $0.3 \%$ \\
\hline & $\mathrm{X} 2 \mathrm{H} 013$ & -25.45 & 30.71 & Krokodil River, Montrose & 1518 & 1959-2012 & 1970-2011 (42 years) & $1.6 \%$ \\
\hline & X2H014 & -25.38 & 30.70 & Houtbosloop, Sudwalaskraal & 250 & $1958-2012$ & 1970-2011 (42 years) & $5.1 \%$ \\
\hline & $\mathrm{X} 2 \mathrm{H} 015$ & -25.49 & 30.70 & Elands River, Lindenau & 1554 & 1959-2012 & 1970-2011 (42 years) & $3.1 \%$ \\
\hline & $\mathrm{X} 2 \mathrm{H} 016$ & -25.36 & 31.96 & Krokodil River, Tenbosch & 10365 & 1960-2012 & 1970-2011 (42 years) & $5.6 \%$ \\
\hline & $\mathrm{X} 2 \mathrm{H} 022$ & -25.54 & 31.32 & Kaap River, Dolton & 1639 & 1960-2012 & 1970-2011 (42 years) & $5.7 \%$ \\
\hline & Х2H024 & -25.71 & 30.84 & Suidkaap River, Glenthorpe & 80 & 1964-2012 & 1970-2011 (42 years) & $1.7 \%$ \\
\hline & $\mathrm{X} 2 \mathrm{H} 031$ & -25.73 & 30.98 & Suidkaap River, Bornmans Drift & 262 & 1966-2012 & 1966-2011 (46 years) & $5.0 \%$ \\
\hline & $\mathrm{X} 2 \mathrm{H} 032$ & -25.51 & 31.22 & Krokodil River, Weltevrede & 5397 & 1968-2012 & 1970-2011 (42 years) & $2.4 \%$ \\
\hline & $\mathrm{X} 2 \mathrm{H} 036$ & -25.44 & 31.98 & Komati River, Komatipoort & 21481 & 1982-2012 & 1983-2011 (28 years) & $4.1 \%$ \\
\hline & X2H046 & -25.40 & 31.61 & Krokodil River, Riverside & 8473 & 1985-2012 & 1986-2011 (26 years) & $2.0 \%$ \\
\hline & $\mathrm{X} 2 \mathrm{H} 047$ & -25.61 & 30.40 & Swartkoppiesspruit, Kindergoed & 110 & $1985-2012$ & 1986-2011 (26 years) & $2.2 \%$ \\
\hline \multirow{9}{*}{ Sabie } & X3H001 & -25.09 & 30.78 & Sabie River, Sabie & 174 & $1948-2012$ & 1970-2011 (42 years) & $0.8 \%$ \\
\hline & X3H002 & -25.09 & 30.78 & Klein Sabie River, Sabie & 55 & 1963-2012 & 1970-2011 (42 years) & $0.4 \%$ \\
\hline & X3H003 & -24.99 & 30.81 & Mac-Mac River, Geelhoutboom & 52 & 1948-2012 & 1970-2011 (42 years) & $0.5 \%$ \\
\hline & Х3H004 & -25.08 & 31.13 & Noordsand River, De Rust & 200 & 1948-2012 & 1970-2011 (42 years) & $3.9 \%$ \\
\hline & Х3H006 & -25.03 & 31.13 & Sabie River, Perry's Farm & 766 & $1958-2000$ & 1970-1999 (30 years) & $2.6 \%$ \\
\hline & Х3H008 & -24.77 & 31.39 & Sand River, Exeter & 1064 & 1967-2011 & 1968-2011 (43 years) & $15.5 \%$ \\
\hline & Х3H011 & -24.89 & 31.09 & Marite River, Injaka & 212 & 1978-2012 & 1979-2011 (32 years) & $7.6 \%$ \\
\hline & X3H015 & -25.15 & 31.94 & Sabie River, Lower Sabie Rest Camp & 5714 & 1986-2012 & 1988-2011 (24 years) & $8.2 \%$ \\
\hline & $\mathrm{X} 3 \mathrm{H} 021$ & -24.97 & 31.52 & Sabie River, Kruger Gate & 2407 & $1990-2012$ & 1991-2011 (21 years) & $10.8 \%$ \\
\hline Lower & E23 & -25.44 & 31.99 & Incomati River, Ressano Garcia & 21200 & 1948-2011 & 1970-2011 (42 years) & $9.0 \%$ \\
\hline Incomati & E43 & -25.03 & 32.65 & Incomati River, Magude & 37500 & 1952-2011 & 1970-2011 (42 years) & $3.5 \%$ \\
\hline
\end{tabular}

Table 4. Hydrologic parameters used in the range of variability approach (Richter et al., 1996).

\begin{tabular}{lll}
\hline $\begin{array}{l}\text { Indicators of hydrologic } \\
\text { alteration group }\end{array}$ & $\begin{array}{l}\text { Regime } \\
\text { characteristics }\end{array}$ & $\begin{array}{l}\text { Hydrological } \\
\text { parameters }\end{array}$ \\
\hline $\begin{array}{l}\text { Group 1: magnitude of monthly water } \\
\text { conditions }\end{array}$ & Magnitude timing & Mean value for each calendar month \\
\hline $\begin{array}{l}\text { Group 2: magnitude and duration of } \\
\text { annual extreme water conditions }\end{array}$ & Magnitude duration & $\begin{array}{l}\text { Annual minima and maxima based on } \\
1,3,7,30 \text { and 90 day means }\end{array}$ \\
\hline $\begin{array}{l}\text { Group 3: timing of annual extreme } \\
\text { water conditions }\end{array}$ & Timing & $\begin{array}{l}\text { Julian date of each annual 1 day } \\
\text { maximum and minimum }\end{array}$ \\
\hline $\begin{array}{l}\text { Group 4: frequency and duration of } \\
\text { high/low pulses }\end{array}$ & Frequency and duration & $\begin{array}{l}\text { No. of high and low pulses each year } \\
\text { Mean duration of high and low pulses } \\
\text { within each year (days) }\end{array}$ \\
\hline $\begin{array}{l}\text { Group 5: rate/frequency of water } \\
\text { condition changes }\end{array}$ & $\begin{array}{l}\text { Rates of change of } \\
\text { frequency }\end{array}$ & $\begin{array}{l}\text { Means of all positive and negative } \\
\text { differences between consecutive daily } \\
\text { values } \\
\text { No. of rises and falls }\end{array}$ \\
\hline
\end{tabular}


Table 5. Description of rainfall stations analysed for trends, the long-term mean annual precipitation (MAP) in mm a ${ }^{-1}$, the standard variation, and the detection of the trend (confidence level of $95 \%$ using the Spearman test) and occurrence change point (using the Pettitt test followed by the $T$ test of stability of the mean and the $F$ test of the stability of variance).

\begin{tabular}{|c|c|c|c|c|c|c|c|c|c|c|}
\hline \multirow[b]{2}{*}{ Name } & \multirow[b]{2}{*}{$\begin{array}{l}\text { Station } \\
\text { ID }\end{array}$} & \multirow[b]{2}{*}{ Latitude } & \multirow[b]{2}{*}{ Longitude } & \multirow[b]{2}{*}{$\begin{array}{l}\text { Altitude } \\
\text { (m a.s.l.) }\end{array}$} & \multirow[b]{2}{*}{$\begin{array}{l}\text { MAP } \\
(\mathrm{mm})\end{array}$} & \multirow[b]{2}{*}{$\begin{array}{r}P \text { reliable } \\
(\%)\end{array}$} & \multicolumn{4}{|c|}{ Analysis for the period 1950 to 2011} \\
\hline & & & & & & & $\begin{array}{r}\text { Mean } \\
\left(\mathrm{mm} \mathrm{a}^{-1}\right)\end{array}$ & $\begin{array}{r}\mathrm{SD} \\
\left(\mathrm{mm} \mathrm{a}^{-1}\right)\end{array}$ & $\begin{array}{l}\text { Trend } \\
\text { Spearman }\end{array}$ & Pettitt \\
\hline Machadodorp & $0517430 \mathrm{~W}$ & -25.67 & 30.25 & 1563 & 781 & 79.6 & 773 & 134 & & \\
\hline Badplaas (Pol) & $0518088 \mathrm{~W}$ & --25.97 & 30.57 & 1165 & 829 & 90.6 & 817 & 153 & & \\
\hline Kaapsehoop & $0518455 \mathrm{~W}$ & -25.58 & 30.77 & 1564 & 1443 & 78.5 & 1461 & 286 & & Decr (1975) \\
\hline Mac-Mac (Bos) & $0594539 \mathrm{~W}$ & -24.98 & 30.82 & 1295 & 1463 & 75.1 & 1501 & 287 & & \\
\hline Spitskop (Bos) & $0555579 \mathrm{~W}$ & -25.15 & 30.83 & 1395 & 1161 & 68.5 & 1197 & 266 & Decr & Decr $(1978)^{*}$ \\
\hline Alkmaar & $0555567 \mathrm{~W}$ & -25.45 & 30.83 & 715 & 830 & 95.2 & 874 & 172 & & \\
\hline Oorschot & $0518859 \mathrm{~W}$ & -25.80 & 30.95 & 796 & 787 & 92.2 & 775 & 185 & & \\
\hline Bosbokrand (Pol) & $0595110 \mathrm{~W}$ & -24.83 & 31.07 & 778 & 982 & 82.4 & 919 & 297 & & Decr $(1978)^{*}$ \\
\hline Pretoriuskop & $0556460 \mathrm{~W}$ & -25.17 & 31.18 & 625 & 707 & 60.0 & 734 & 188 & & \\
\hline Riverbank & $0519310 \mathrm{~W}$ & -25.67 & 31.23 & 583 & 683 & 70.5 & 782 & 163 & Incr & $\operatorname{Incr}(1977)^{* *}$ \\
\hline Piggs Pig & 0519448 A & -25.97 & 31.25 & 1029 & 1024 & 40.1 & 1075 & 315 & Decr & Decr $(1978)^{*}$ \\
\hline Skukuza & $0596179 \mathrm{~W}$ & -25.00 & 31.58 & 300 & 560 & 63.1 & 566 & 140 & & \\
\hline Riverside & $0557115 \mathrm{~W}$ & -25.42 & 31.60 & 315 & 547 & 66.5 & 520 & 187 & & \\
\hline Satara & $0639504 \mathrm{~W}$ & -24.40 & 31.78 & 257 & 568 & 42.1 & 602 & 151 & Incr & Incr (1971) \\
\hline Fig Tree & $0520589 \mathrm{~W}$ & -25.82 & 31.83 & 256 & 591 & 63.4 & 594 & 145 & Decr & Decr $(1978)^{*}$ \\
\hline Tsokwane & $0596647 \mathrm{~W}$ & -24.78 & 31.87 & 262 & 540 & 66.1 & 544 & 134 & & $\operatorname{Incr}(1971)^{*}$ \\
\hline Krokodilbrug & $0557712 \mathrm{~W}$ & -25.37 & 31.90 & 192 & 584 & 62.9 & 590 & 147 & & \\
\hline Moamba & P821 M & -25.60 & 32.23 & 108 & 632 & 63.9 & 633 & 185 & & \\
\hline Xinavane & P10 M & -25.07 & 32.87 & 18 & 853 & 76.2 & 773 & 241 & & \\
\hline Manhica & P63 M & -25.40 & 32.80 & 33 & 883 & 86.2 & 903 & 275 & & $\operatorname{Incr}(1970)^{* *}$ \\
\hline
\end{tabular}

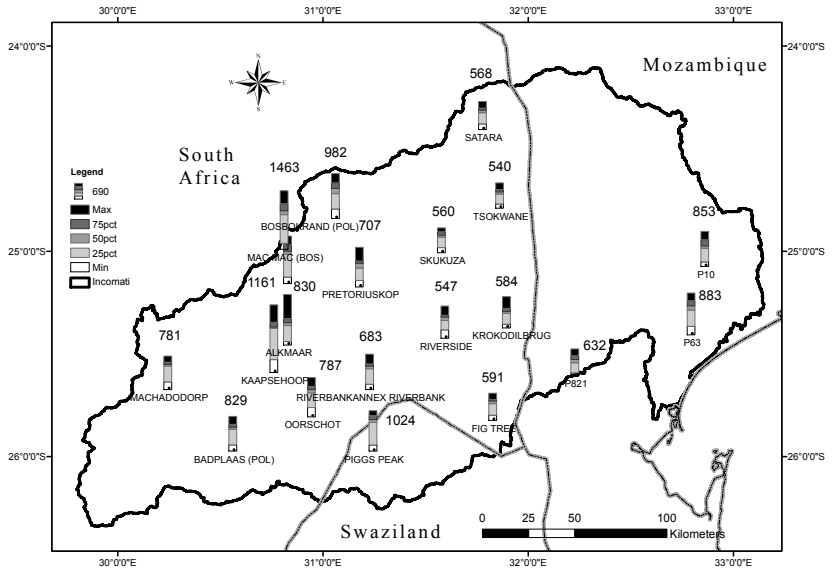

Figure 3. Map illustrating the spatial variation of annual rainfall across the Incomati basin (minimum, $25 \%$, median, $75 \%$, and maximum annual precipitation are shown in the bar; the number on top of the bar is the Mean Annual Precipitation for the period 19502000).

\section{Results}

\subsection{Rainfall}

Data series of 20 rainfall stations were statistically analysed for the period 1950-2011 (Table 5). The variability of rainfall across the basin was confirmed to be high, both intraand inter-annually, with a wide range between years. This variability is highest for the stations located in mountainous areas. The variability across the basin is also significant, as illustrated by the boxplot of Fig. 3 .

The Spearman trend test revealed that only 5 of the 20 investigated stations showed significant trends of increase (2 stations) and decrease ( 3 stations). However, the stations that presented significant trends are also stations with a lower percentage of reliability; thus, it is possible that the trend identified could be affected by data infilling procedures. There was no serial correlation of annual and monthly time series. Some change points were identified using the Pettitt test, mostly in the years 1971 and 1978 (Table 5). Only 2 stations out of the 20 studied showed significant change towards a wetter regime (Riverbank and Manhica).

Monthly rainfall also does not exhibit any clear trend at most stations. This is consistent with the larger-scale analyses conducted by Schulze (2012) for South Africa and Shongwe et al. (2009) for southern Africa. 
Table 6. Hydrological indicators of main sub-catchments.

\begin{tabular}{|c|c|c|c|c|c|c|c|c|c|c|c|}
\hline \multicolumn{2}{|l|}{$\begin{array}{l}\text { Streamflow } \\
\text { indicators }\end{array}$} & \multicolumn{2}{|c|}{$\begin{array}{c}\text { Komati } \\
\text { X1H003 - Tonga }\end{array}$} & \multicolumn{2}{|c|}{$\begin{array}{c}\text { Crocodile } \\
\text { X2H016 - Tenbosh }\end{array}$} & \multicolumn{2}{|c|}{$\begin{array}{c}\text { Incomati } \\
\mathrm{X} 2 \mathrm{H} 036 \text { - Komatipoort }\end{array}$} & \multicolumn{2}{|c|}{$\begin{array}{c}\text { Sabie } \\
\text { X3H015 - Lower Sabie }\end{array}$} & \multicolumn{2}{|c|}{$\begin{array}{c}\text { Incomati } \\
\text { E43 - Magude }\end{array}$} \\
\hline \multirow{3}{*}{$\begin{array}{l}\text { Period of } \\
\text { analysis: } \\
\text { Drainage area }\end{array}$} & \multirow{3}{*}{$\begin{array}{r}\text { Units } \\
\mathrm{km}^{2}\end{array}$} & \multirow{2}{*}{\multicolumn{2}{|c|}{$\begin{array}{l}1970-2011 \\
(42 \text { years }) \\
8614\end{array}$}} & \multicolumn{2}{|c|}{$\begin{array}{l}1970-2011 \\
(42 \text { years })\end{array}$} & \multicolumn{2}{|c|}{$\begin{array}{l}1983-2011 \\
(28 \text { years })\end{array}$} & \multicolumn{2}{|c|}{$\begin{array}{l}1988-2011 \\
(24 \text { years })\end{array}$} & \multicolumn{2}{|c|}{$\begin{array}{l}1970-2011 \\
(42 \text { years })\end{array}$} \\
\hline & & & & 10365 & & 21481 & & 5714 & & 37500 & \\
\hline & & Median & $\mathrm{CD}^{* *}$ & Median & $\mathrm{CD}^{* *}$ & Median & $\mathrm{CD}^{* *}$ & Median & $\mathrm{CD}^{* *}$ & Median & $\mathrm{CD} * *$ \\
\hline Annual* & $\mathrm{m}^{3} \mathrm{~s}^{-1}$ & 16.94 & 2.14 & 21.35 & 1.97 & 34.28 & 2.11 & 17.35 & 2.31 & 47.44 & 2.01 \\
\hline October & $\mathrm{m}^{3} \mathrm{~s}^{-1}$ & 3.95 & 1.47 & 2.54 & 1.88 & 2.24 & 1.87 & 3.08 & 0.92 & 8.72 & 1.21 \\
\hline November & $\mathrm{m}^{3} \mathrm{~s}^{-1}$ & 5.72 & 1.94 & 5.75 & 2.35 & 7.09 & 3.88 & 4.81 & 1.09 & 16.14 & 1.49 \\
\hline December & $\mathrm{m}^{3} \mathrm{~s}^{-1}$ & 11.46 & 2.09 & 15.07 & 1.48 & 18.79 & 2.63 & 10.83 & 1.49 & 22.91 & 2.90 \\
\hline January & $\mathrm{m}^{3} \mathrm{~s}^{-1}$ & 17.26 & 1.82 & 20.68 & 1.47 & 34.47 & 1.52 & 18.52 & 1.35 & 37.96 & 1.35 \\
\hline February & $\mathrm{m}^{3} \mathrm{~s}^{-1}$ & 25.09 & 1.95 & 31.37 & 2.01 & 29.77 & 2.80 & 16.33 & 1.84 & 45.09 & 3.21 \\
\hline March & $\mathrm{m}^{3} \mathrm{~s}^{-1}$ & 18.33 & 1.74 & 27.15 & 1.63 & 42.15 & 1.90 & 19.51 & 2.30 & 51.75 & 2.32 \\
\hline April & $\mathrm{m}^{3} \mathrm{~s}^{-1}$ & 11.64 & 1.74 & 19.82 & 1.37 & 24.10 & 2.13 & 13.69 & 1.13 & 34.90 & 2.03 \\
\hline May & $\mathrm{m}^{3} \mathrm{~s}^{-1}$ & 8.03 & 1.41 & 9.11 & 1.68 & 9.98 & 2.16 & 7.04 & 1.64 & 17.85 & 1.86 \\
\hline June & $\mathrm{m}^{3} \mathrm{~s}^{-1}$ & 4.96 & 1.90 & 5.66 & 1.62 & 7.10 & 2.45 & 5.64 & 1.25 & 14.04 & 1.44 \\
\hline July & $\mathrm{m}^{3} \mathrm{~s}^{-1}$ & 3.77 & 1.98 & 4.56 & 1.48 & 4.72 & 2.28 & 3.79 & 1.18 & 10.41 & 1.47 \\
\hline August & $\mathrm{m}^{3} \mathrm{~s}^{-1}$ & 2.67 & 1.63 & 2.63 & 1.71 & 2.51 & 1.35 & 3.40 & 1.08 & 8.46 & 1.41 \\
\hline September & $\mathrm{m}^{3} \mathrm{~s}^{-1}$ & 2.43 & 1.47 & 2.08 & 1.81 & 2.24 & 1.51 & 2.69 & 1.15 & 7.06 & 1.11 \\
\hline 1 day minimum & $\mathrm{m}^{3} \mathrm{~s}^{-1}$ & 0.31 & 4.04 & 0.24 & 2.64 & 0.14 & 5.29 & 1.45 & 1.13 & 2.49 & 1.48 \\
\hline 3 day minimum & $\mathrm{m}^{3} \mathrm{~s}^{-1}$ & 0.38 & 3.38 & 0.32 & 2.16 & 0.25 & 3.76 & 1.53 & 1.08 & 2.71 & 1.76 \\
\hline 7 day minimum & $\mathrm{m}^{3} \mathrm{~s}^{-1}$ & 0.59 & 2.55 & 0.40 & 2.88 & 0.33 & 4.35 & 1.60 & 1.16 & 3.01 & 1.61 \\
\hline 30 day minimum & $\mathrm{m}^{3} \mathrm{~s}^{-1}$ & 1.46 & 2.13 & 1.52 & 1.79 & 1.29 & 2.08 & 2.01 & 1.12 & 4.84 & 1.37 \\
\hline 90 day minimum & $\mathrm{m}^{3} \mathrm{~s}^{-1}$ & 3.69 & 1.47 & 3.45 & 1.34 & 3.17 & 2.09 & 3.02 & 1.23 & 8.14 & 1.38 \\
\hline 1 day maximum & $\mathrm{m}^{3} \mathrm{~s}^{-1}$ & 134.4 & 1.26 & 142.2 & 1.38 & 274.3 & 1.00 & 113 & 2.51 & 381.5 & 1.80 \\
\hline 3 day maximum & $\mathrm{m}^{3} \mathrm{~s}^{-1}$ & 102.9 & 1.50 & 126.9 & 1.33 & 232.9 & 1.15 & 87.62 & 2.60 & 344.1 & 1.74 \\
\hline 7 day maximum & $\mathrm{m}^{3} \mathrm{~s}^{-1}$ & 81.79 & 1.59 & 107.4 & 1.20 & 201.4 & 1.13 & 62.55 & 2.27 & 273.7 & 1.56 \\
\hline 30 day maximum & $\mathrm{m}^{3} \mathrm{~s}^{-1}$ & 54.39 & 1.45 & 76.98 & 1.28 & 109.6 & 1.33 & 37.66 & 1.93 & 156.7 & 1.45 \\
\hline 90 day maximum & $\mathrm{m}^{3} \mathrm{~s}^{-1}$ & 39.19 & 1.33 & 45.08 & 1.16 & 68.69 & 1.71 & 28.06 & 1.47 & 102 & 1.32 \\
\hline Date of & Julian & & & & & & & & & & \\
\hline minimum & Date & 275 & 0.10 & 274 & 0.12 & 281.5 & 0.15 & 278.5 & 0.06 & 290.5 & 0.21 \\
\hline Date of & Julian & & & & & & & & & & \\
\hline maximum & Date & 38.5 & 0.16 & 33 & 0.11 & 35.5 & 0.19 & 20.5 & 0.17 & 39.5 & 0.14 \\
\hline Low pulse count & No. & 6 & 1.63 & 4 & 1.63 & 5 & 1.55 & 4 & 1.00 & 3 & 1.33 \\
\hline Low pulse duration & Days & 5.5 & 1.41 & 5 & 1.60 & 3.5 & 0.71 & 6.5 & 1.69 & 6.75 & 2.09 \\
\hline High pulse count & No. & 6 & 0.75 & 4 & 1.25 & 5 & 0.95 & 4 & 0.69 & 4 & 0.75 \\
\hline High pulse duration & Days & 4 & 1.31 & 4 & 2.13 & 4.5 & 1.28 & 5 & 2.10 & 8.5 & 1.03 \\
\hline Rise rate & $\mathrm{m}^{3} \mathrm{~s}^{-1}$ & 0.7095 & 1.39 & 0.64 & 0.98 & 1.161 & 1.38 & 0.404 & 1.12 & 1.058 & 1.43 \\
\hline Fall rate & $\mathrm{m}^{3} \mathrm{~s}^{-1}$ & -0.7295 & -0.98 & -0.61 & -0.78 & -1.38 & -1.28 & -0.2398 & -1.10 & -0.6278 & -2.31 \\
\hline $\begin{array}{l}\text { Number of } \\
\text { reversals }\end{array}$ & No & 111.5 & 0.26 & 113 & 0.42 & 121 & 0.18 & 95 & 0.29 & 86 & 0.49 \\
\hline
\end{tabular}

* In the annual statistics, the mean and coefficient of variation were used. ${ }^{* *} \mathrm{CD}$ is the coefficient of dispersion. More details about $\mathrm{CD}$ are available in the text.

Mussá et al. (2013) studied the trends of annual and dry extreme rainfall using the Standardized Precipitation Index (SPI), and also found no significant trends in annual rainfall extremes across the Crocodile sub-catchment.

\subsection{Variability of streamflow}

The metrics of the different hydrologic indicators were compiled as an output of the IHA analysis, which is illustrated for the gauging stations located at the outlet (or the most downstream) of each main sub-catchment in Table 6. The variability is described, using non-parametric statistics (median and coefficient of dispersion), because the hydrological time se- ries are not normally distributed, but are positively skewed. The coefficient of dispersion $(\mathrm{CD})$ is defined as $\mathrm{CD}=(75 \mathrm{th}$ percentile -25 th percentile)/50th percentile. The larger the $\mathrm{CD}$, the larger the variation of the parameter will be.

The flow patterns are consistent with the summer rainfall regime, with the highest flow and rainfall events associated with tropical cyclone activity in January-March.

A comparison of the flow normalised by area (Fig. 4) for the main sub-catchments reveals that the Sabie yields a higher runoff than the Komati and the Crocodile. This is the case because the observed streamflows include the impact of water abstractions and streamflow reduction activities 


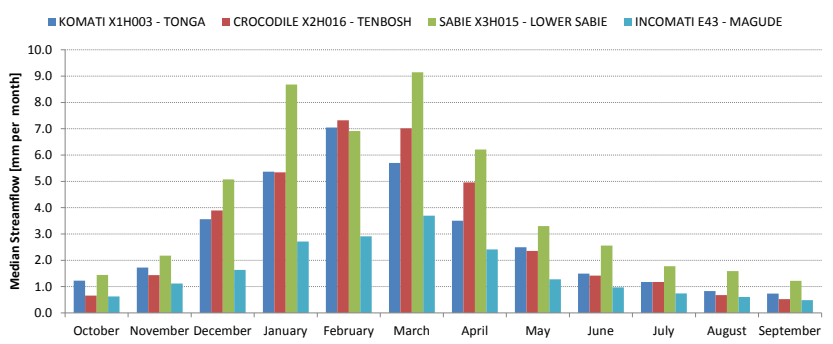

Figure 4. Median of observed daily streamflow for the gauges located at the outlets of the major sub-catchments: Komati, Crocodile, Lower Sabie and Incomati (based on daily time series from 1970 to 2011).

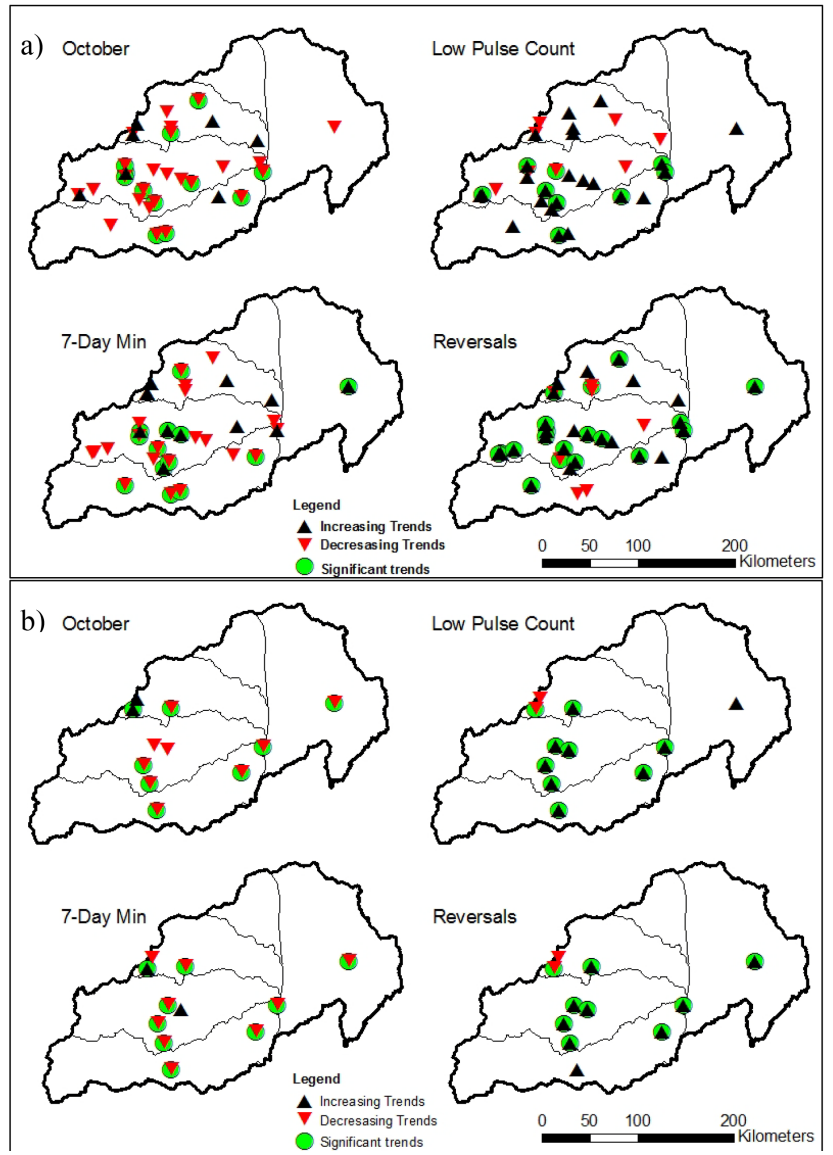

Figure 5. Trends of different indicators of streamflow: (a) for period 1970-2011; (b) for period 1950-2011.

(Hughes and Mallory, 2008; DWAF, 2009a), which are more intense in the Komati and Crocodile sub-catchments.

Another aspect to note is that the flows of February are likely to be higher than observed records, but high streamflow extremes are not fully captured by the current monitoring network, due to gauge limits.

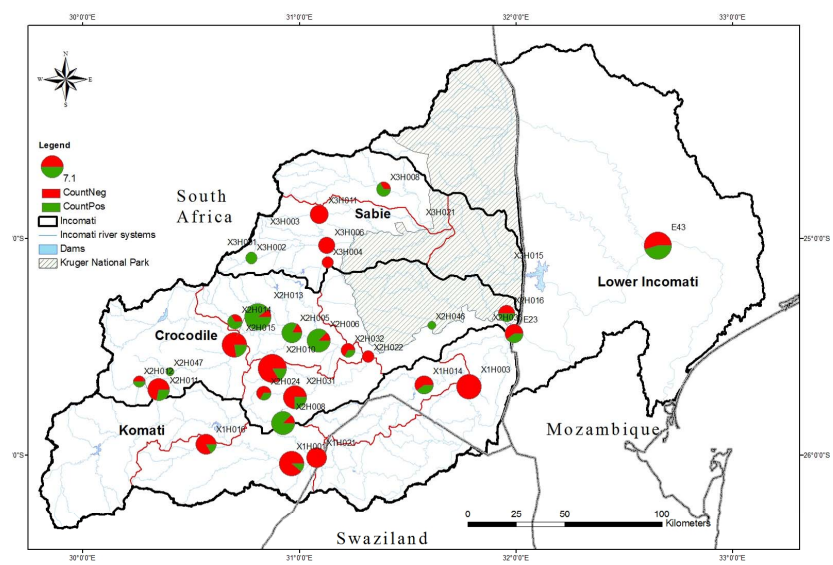

Figure 6. Count of significant trends. Declining trends are in red and increasing trends in green. The size of the pie is proportional to the total number of significant trends.

\subsection{Trends in streamflow}

Figure 5 presents a spatial plot of trends for selected hydrological indicators for the periods 1970-2011 (Fig. 5a) and 1950-2011 (Fig. 5b). The significant trends are highlighted with a circle. Table 7 presents the slope of the trend lines and $P$ values for the gauges located at the outlet, or the most downstream point of each main sub-catchment. There is a significant trend of decreasing mean flow in October at almost all stations, especially the ones located on the main stem of the Crocodile and Komati rivers. October is the month of the start of the rainy season, when the dam levels are lowest and irrigation water requirements highest (DWAF, 2009a; ICMA, 2010).

This trend is consistent with the decreasing trends of minimum flows, as exemplified by the 7 day minimum. In contrast, it can be seen that the count of low pulses increased significantly in many gauges, which indicates the more frequent occurrence of low flows. Another striking trend is the significant increase in the number of reversals at almost all stations. Reversals are calculated by dividing the hydrologic record into "rising" and "falling" periods, which correspond to periods in which daily changes in flows are either positive or negative, respectively. The number of reversals is the number of times that the flow switches from one type of period to another. The observed increased number of reversals is likely due to the effect of flow regulation and water abstractions.

The significant trends (95\% confidence level) of the various indicators were counted per station and plotted on a map (Fig. 6). Most significant decreasing trends occur in the Komati and Crocodile systems, which are also the most stressed sub-catchments. An interesting aspect is that some of the trends cross-compensate each other. Some of the positive trends occurring in the tributaries of the Crocodile, for example, the October median flow and the baseflow, are cancelled out when moving down the main stem of the river. 
Table 7. Trends of the hydrological indicators for the period 1970-2011. In bold are significant trends at the $95 \%$ confidence level.

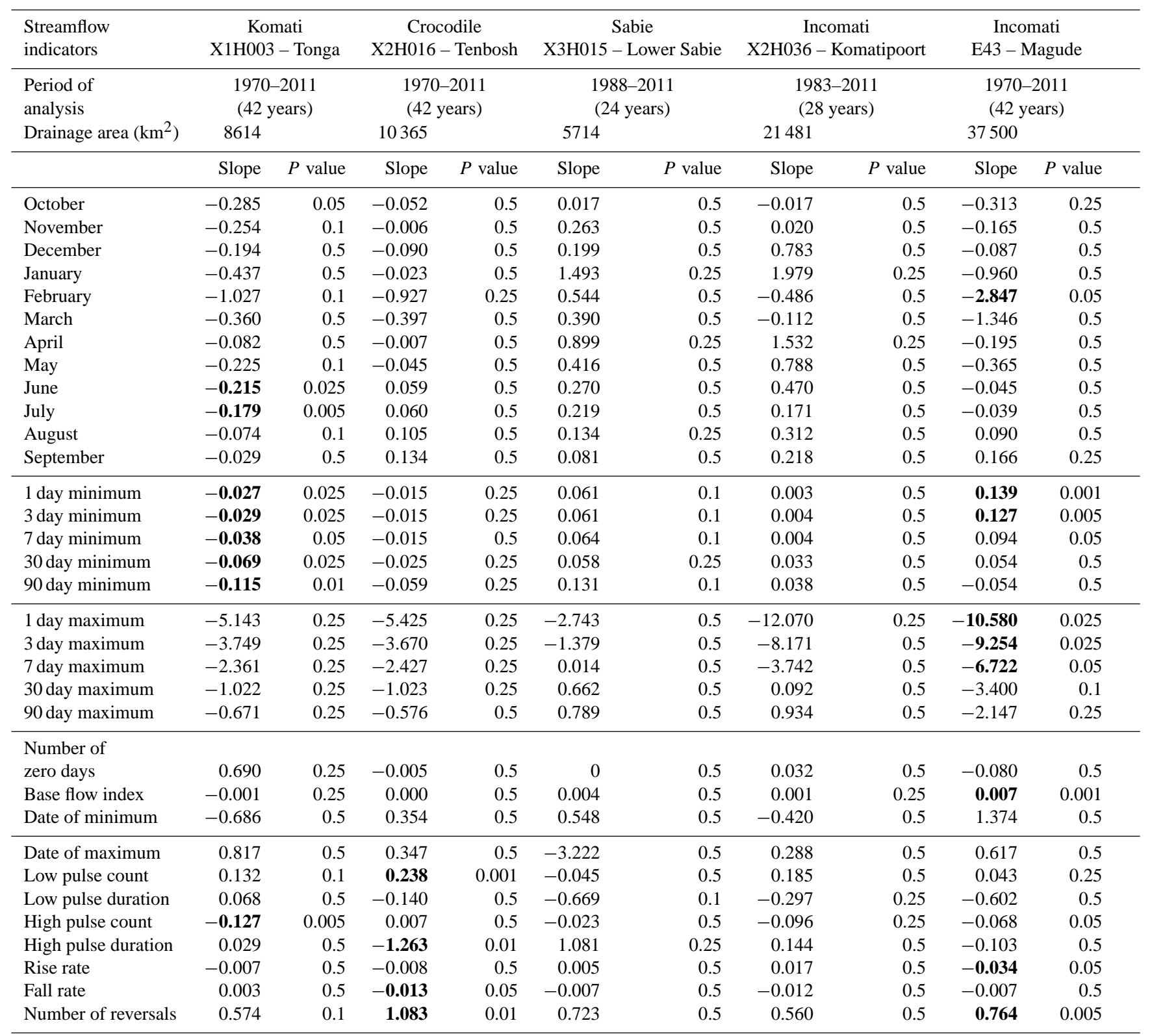

The cross-compensation can also be observed on a basin scale in the Sabie, where the trends of decreasing flows are not so frequent or significant. It is likely that this occurs because the majority of the Sabie falls under the conservation area of the Kruger National Park (KNP), and therefore fewer abstractions occur compared to other sub-catchments, as illustrated in Table 1. The KNP has been playing an important role in the catchment management fora set up by the Inkomati Catchment Management Agency (ICMA), which concern the provision of environmental minimum flows, in order to maintain ecosystem services and biodiversity in the park (Pollard et al., 2012; Riddell et al., 2013).
Table 7 illustrates that many of the trends observed in the Sabie sub-catchment contrast with those observed in the Komati and Crocodile sub-catchments. Thus, the trends observed in downstream Magude (station E43) in Mozambique are the result of a combination of the positive effect of the conservation approach of KNP on the Sabie, and the negative effect of flow reductions on the Crocodile and the Komati.

The Komati sub-catchment (at Tonga gauge, X1H003) is where most negative trends occur, particularly significant during the months of October, June and July (Table 7 and Fig. 6). At the downstream end of the Crocodile (at Tenbosch gauge, X2H016), similar trends are not visible, because of cross-compensations: the Kaap and Elands tribu- 


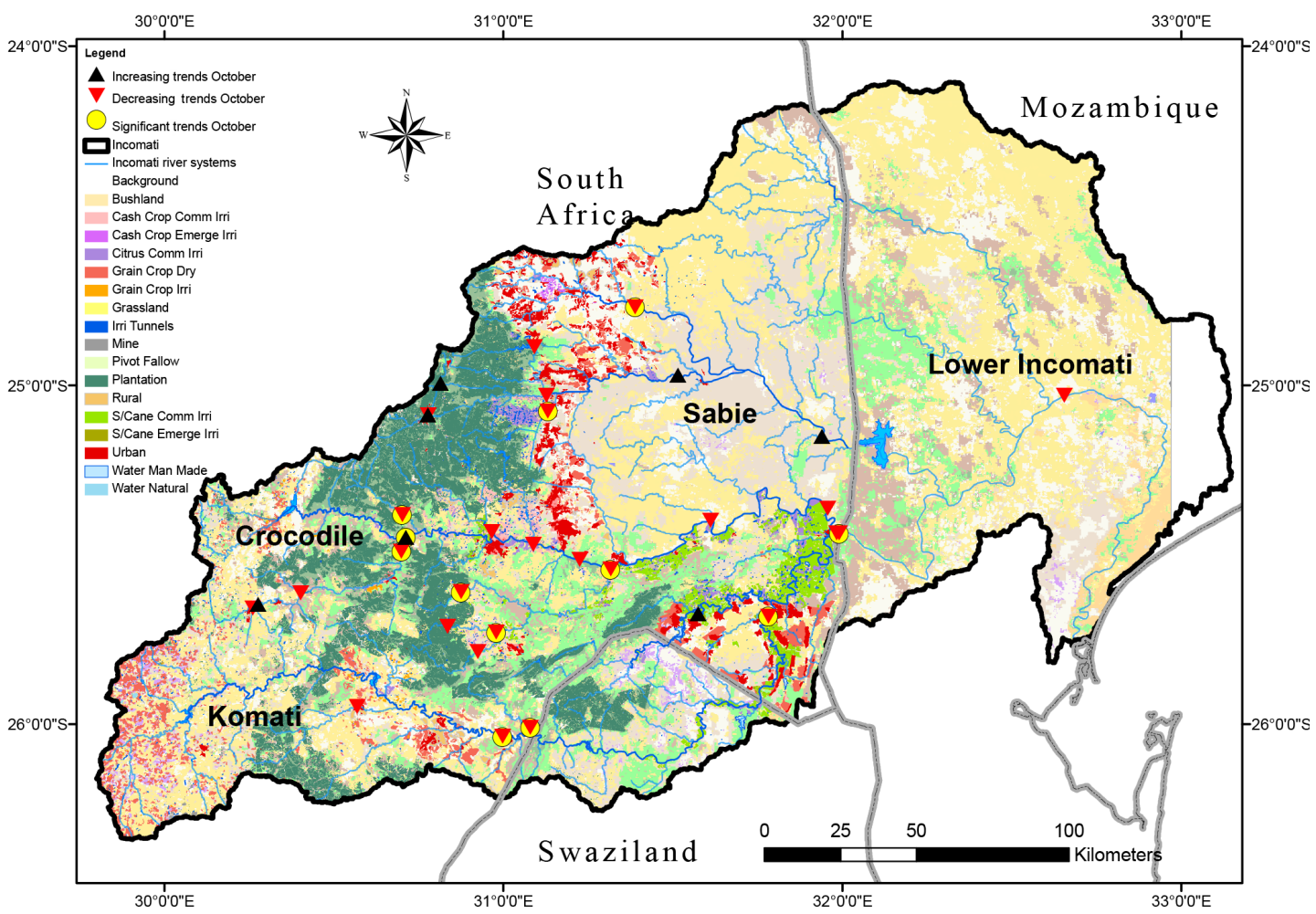

Figure 7. Land-use land-cover map of Incomati (ICMA, 2010; TPTC, 2010) and streamflow trends in the month of October.

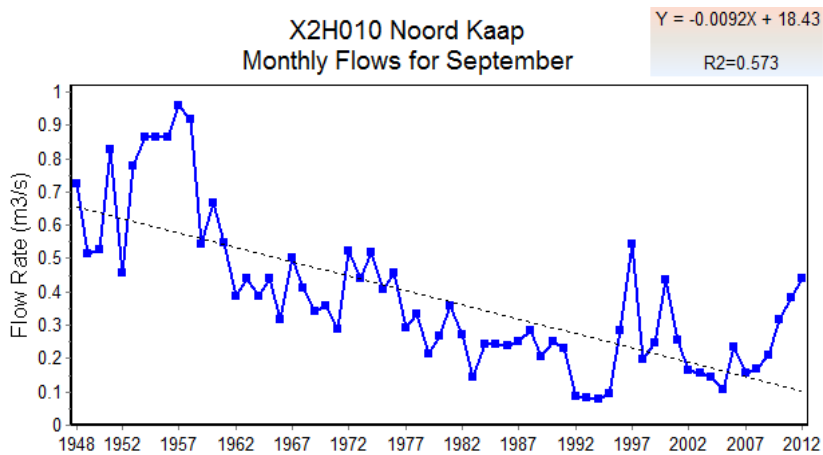

Figure 8. Plot of median monthly flows for September for the entire time series (1949-2011) on the Noord Kaap gauge, located in the Crocodile sub-catchment.

taries both have significant decreasing trends of their mean monthly flows, as well as the low flows; the Kwena Dam, located on the main stem of the Crocodile, on the other hand, is managed in a way so as to augment the flows during the dry season.

It is important to note that these trends are even more pronounced when longer time series are considered. Two examples from the Crocodile basin are presented below.

\subsubsection{Example of decreasing trends: Noord Kaap X2H010}

The Noord Kaap gauge (X2H010), located on a tributary of the Crocodile sub-catchment, displays the most intriguing trends. Out of the 33 IHA indicators, this gauge had 12 significant trends, 10 of them negative, indicating a major shift in flow regime. The decreasing trends occur in all months, but are more pronounced during low flow months, particularly September (Fig. 8) and October. There is a significant decrease in high flows and small floods and an increase in extreme low flows. However, there is no record of the presence of a dam or major infrastructure (DWAF, 2009d). The areal rainfall for the drainage area of this gauge does not show a significant decreasing trend, which suggests that the reduction observed in streamflow should be a result of land-use change, namely, conversion to forestry and irrigated land. Fig. 9 illustrates the comparison of median monthly flows for the two periods. From the analysis of land-use changes over time (Table 2), it can be seen that the sharp decrease in mean monthly flows during the 1960 s coincides with an increase in the area under irrigated agriculture. During the 1970s, there was also a great increase in area under forestry, namely, eucalyptus (DWAF, 2009a). Commercial forestry consumes more water through evaporation than the native vegetation it replaces; therefore, under the South African National Water Act, it must be licensed as a water user, which is termed a 


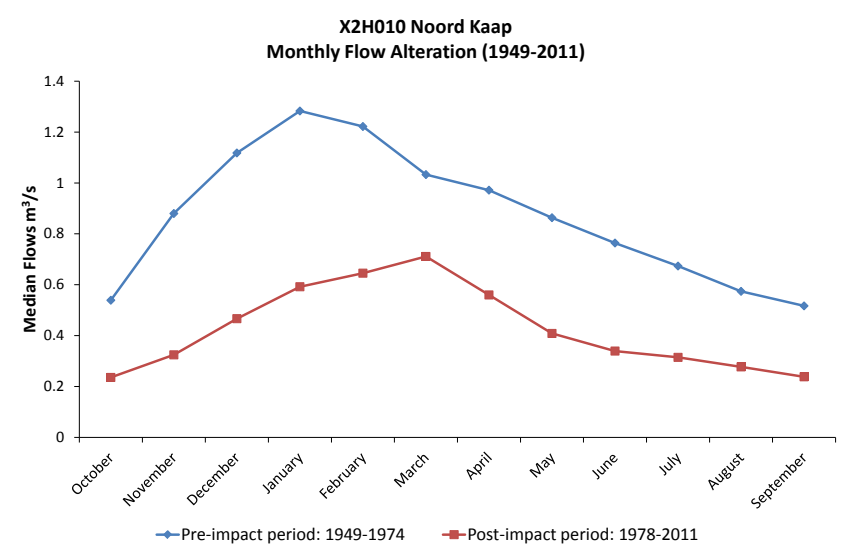

Figure 9. Plot of median monthly flows for two periods (1949-1974 and 1978-2011) on the Noord Kaap gauge, located in the Crocodile sub-catchment.

streamflow reduction activity (SFRA) (Jewitt, 2002, 2006b). A recent study by van Eekelen et al. (2015) finds that streamflow reduction due to forest plantations may be 2 or even 3 times more than that allowed by the Interim IncoMaputo Agreement.

\subsubsection{Impact of the Kwena Dam on streamflows of the Crocodile River}

The Kwena Dam is the main reservoir in the Crocodile system, located upstream in the catchment and commissioned in 1984. The dam is used to improve the assurance of supply of water for irrigation purposes in the catchment. The Montrose gauge (X2H013) is located a few kilometres downstream of this dam. The two-period (1959-1984 and 1986-2011) analysis illustrates the main impacts of the Kwena Dam on the river flow regime, namely the dampening of peak flows and an increase in low flows (Fig. 10). These results are consistent with the analysis conducted by Riddell et al. (2013), which found significant alterations of the natural flow regime in the Crocodile basin over the past 40 years. Similar impacts were found in studies in different parts of the world (Richter et al., 1998; Bunn and Arthington, 2002; Maingi and Marsh, 2002; Birkel et al., 2014).

It can be seen that this reservoir is managed to augment the low flows and attenuate floods. This change in the flow regime influences the streamflow along the main stem of the Crocodile River, but as tributaries join and water is abstracted, the effect is reduced. At the outlet at Tenbosch gauge X2H016 (Fig. 6 and Table 7), the effects of flow regulation and water abstractions have counter-balanced the contrasting trends observed upstream.

\subsubsection{Impact of anthropogenic actions}

As can be seen from water-use information, the impacts of land-use change and water abstractions are the main drivers

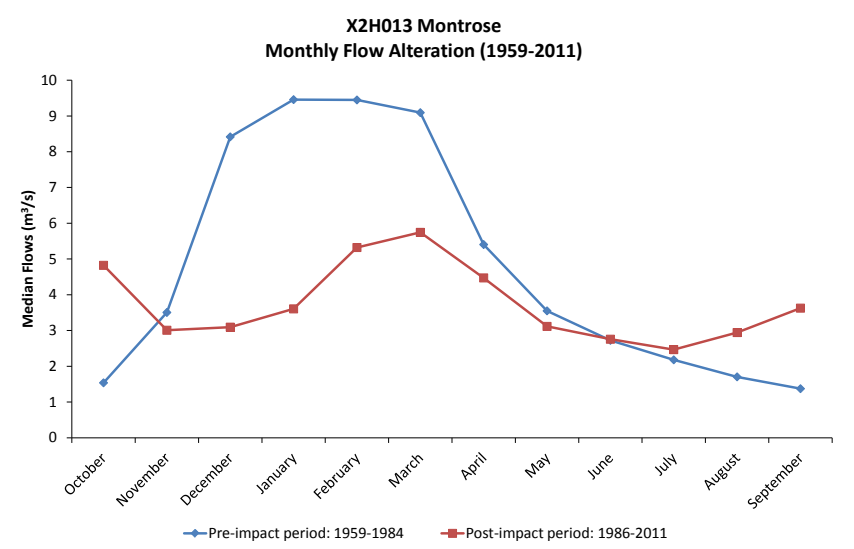

Figure 10. Impact of the Kwena Dam (commissioned in 1984) on streamflows of the Crocodile River, Montrose gauge X2H013.

of changes in the flow regime on the Incomati. However, the situation is variable along the catchment. In the Sabie system, in spite of large areas of commercial forestry in the headwaters, the indicators of mean, annual and low flows do not show significant trends (Table 7). This can be explained by the fact that most of the forestry area was already established during the period of analysis (1970-2011) (DWAF, 2009c). The fact that a large proportion of the Sabie sub-catchment is under conservation land use (KNP and other game reserves) also plays an important role in maintaining the natural flow regime.

On the Crocodile, however, irrigated agriculture, forestry and urbanisation were the most important anthropogenic drivers. They affect the streamflow regime, the water quantity and possibly the water quality as well (beyond the scope of this analysis). This has important implications when environmental flow requirements and minimum cross-border flows need to be adhered to. Pollard and du Toit (2011a) and Riddell et al. (2013) have demonstrated that the Crocodile River is not complying with environmental flow requirements during most of the dry season at the outlet.

On the Komati, the strategic water uses, which have first priority (such as the water transfers to ESKOM plants in the Olifants catchment and to irrigation schemes in the Umbeluzi) (Nkomo and van der Zaag, 2004; DWAF, 2009b), have a high impact on streamflows. Because of other water allocations, for irrigation, forestry and other industries, steady trends of decreasing flows could be identified. This is another system where the environmental flows and crossborder requirements are often not met during the dry season (Pollard and du Toit, 2011a; Mukororira, 2012; Riddell et al., 2013). 


\section{Discussion}

\subsection{Limitations of this study}

The available data series have some gaps, especially during high flow periods. Because of this, the analysis of high flow extremes is highly uncertain. For the trend analysis, the period of common data followed the construction of several impoundments and other developments.

Another challenge is the disparity of data availability across the different riparian countries. In Mozambique, only two gauges had reliable flow data for this analysis, representing the entire lower Incomati system. The rivers Massintoto, Uanetse and Mazimechopes in Mozambique do not have active flow gauges. There is definitely a need to strengthen the hydrometric monitoring network in the Mozambican part of the basin, as well as in the tributaries originating in the Kruger National Park.

\subsection{What are the most striking trends and where do they occur?}

The analysis resulted in the identification of major trends, including

- decreasing trends in the magnitude of monthly flow (significant for low flow months, e.g. October), minimum flow (1, 3, 7, 30 and 90 day minima) and the occurrence of high flow pulses,

- significant increasing trends of the magnitude of monthly flow (August and September) in some locations in the Crocodile and Sabie, and in the occurrence of flow reversals basin-wide, and

- Some gauges showed no significant change or no clear pattern of change in the parameters analysed. These are mainly gauges located on the Sabie, which by 1970 had already established the current land use.

In the Komati system, the flow regulation and water abstractions have strong impacts on streamflow. Most gauges are severely impacted and it is quite difficult to characterise natural flow conditions. Flow regulation has the largest impact on low flow and minimum flows. In the Komati, irrigated agriculture is significant, particularly sugarcane. The upstream dams of Nooitgedacht and Vygeboom are mainly used to supply cooling water to ESKOM power stations outside the basin; thus, this water is exported and not used within the basin.

In the Crocodile system, flow regulation by the Kwena Dam has attenuated extreme flow events. The high flows are reduced and the low flows generally increase, leading to reverse seasonality downstream. Reverse seasonality is the change in timing of hydrograph characteristics, for example, the occurrence of low flows in the wet season or high flows in the dry season. The Kwena Dam is used to improve the assurance of the supply of water for irrigation purposes in the catchment. However, Noord Kaap gauge X2H010, a headwater tributary of the Crocodile system, experiences a significant and dramatic reduction in flows, shown in the monthly flow, and the low flow parameters. This change was compared with the increase in the area under forestry in the sub-catchment, as well as with the increase in irrigation. The comparison revealed that the land-use change was the main driver of the flow alteration.

In the Sabie system, most gauges did not show significant trends. This is most likely due to fewer disturbances compared to the other main catchments: lower water demands, fewer water abstractions and larger areas under conservation. During the period 1970-2011 there were no clear impacts of climatic change (in terms of rainfall) on streamflow.

\subsection{Implications of these findings for water resource management}

The results of this study illustrate some hotspots where more attention should be put in order to ensure provision of water to society and the environment. When the analysis of trends is combined with the land use of the basin (Fig. 7), it is clear that the majority of gauges with decreasing trends are located in areas where forestry or irrigated agriculture dominates the land use and where conservation approaches are less prevalent. The presence of water management infrastructure (dams) greatly influences the flow regime.

For the management of water resources in the basin, it is important to note some clear patterns, illustrated by the Sabie, Crocodile and Komati. The Sabie flows generated in the upper parts of the catchment persist until the outlet, whilst in other rivers, flows are highly modified. This suggests that the use of the conservation approach through the strategic adaptive management of the Kruger National Park (KNP) and the Inkomati Catchment Management Agency (ICMA), which are stronger on the Sabie, can be very beneficial for keeping environmental flows in the system. It is important to consider not only the magnitude of flows, but their duration and timing as well.

Dams provide storage, generate hydropower and attenuate floods in the basin, but have impacts downstream, such as the change in mean monthly flows, the reversal of seasonality and the trapping of sediments, which can all hamper the health of downstream ecosystems. The recently concluded Mbombela Reconciliation Strategy (Beumer and Mallory, 2014) strongly recommends the construction of new dams in South Africa, including one at Mountain View in the Kaap sub-catchment. The plans for these developments happen when Swaziland is not yet fully utilising its allocation under the Piggs Peak Agreement and the Interim IncoMaputo Agreement (TPTC, 2010). Experiences of other countries around the world show that dam construction has many, often wide-ranging and long-term, social and ecological impacts that often are negative and that frequently are 
irreversible, including the social upheaval caused by the resettlement of communities, loss of ecosystems and biodiversity, increased sediment trapping, irreversible alteration of flow regimes and the prohibitive cost of decommissioning (see, for an overview, Tullos et al., 2009, and Moore et al., 2010). It is therefore important to explore alternative options fully before deciding on the construction of more large dams. So, alternative possibilities of restoring natural stream flows and/or increasing water storage capacity should be investigated further and adopted. These alternatives could include aquifer storage, artificial recharge, rainfall harvesting, decentralised storage, and reducing the water use of existing uses and users, including irrigation, industry and forest plantations. The operation rules of existing and future dams should also include objectives to better mimic crucial aspects of the system's natural variability.

Given the likely expansion of water demands due to urbanisation and industrial development, it is also important that water demand management and water conservation measures are better implemented in the basin. For example, there could be systems to reward users that use technology to improve their water-use efficiency and municipalities that encourage their users to lower water use.

This study also shows the complexity of water resource availability and variability. The complexity is even more relevant, considering that this is a trans-boundary basin and that there are international agreements regarding minimum crossborder flows and maximum development levels that have to be adhered to (Nkomo and van der Zaag, 2004; Pollard and Toit, 2011a, b; Riddell et al., 2013).

There is a great discrepancy in data availability between different riparian countries. It is important that Mozambique, in particular, improves its monitoring network, in order better to assess the impact of various management activities occurring upstream on the state of water resources. The monitoring of hydrological extremes should receive more attention, with the focus on increasing the accuracy of recording the flood events. The improvement of the monitoring network can be achieved by various means, such as

- water management institutions collaborating more intensely with academic and consultant institutions,

- developing realistic plans to improve monitoring and data management,

- learning from other countries/institutions that have adequate monitoring in place,

- using modern ICT and other technologies, which may become cheaper and more accessible, and

- involving more stakeholders and citizens in data collection.

\section{Conclusions}

The research conducted reveals the dynamics of streamflow and their drivers in a river basin.

The statistical analysis of rainfall data revealed no consistent significant trend of increase or decrease for the studied period. The analysis of streamflow, on the other hand, revealed significant decreasing trends of streamflow indicators, particularly the median monthly flows of September and October, and low flow indicators. This study concludes that land use and flow regulation are the largest drivers of streamflow trends in the basin. Indeed, over the past 40 years, the areas under commercial forestry and irrigated agriculture have increased over 4 times, increasing the consumptive water use basin-wide.

The study therefore recommends that the strategic adaptive management adopted by the Kruger National Park and the Inkomati Catchment Management Agency should be employed further in the basin. Water demand management and water conservation should be alternative options to the development of dams, and should be investigated further and established in the basin. Land-use practices, particularly forestry and agriculture, have a significant impact on the water quantity of the basin; therefore, stakeholders from these sectors should work closely with the water management institutions when planning for future developments and water allocation plans.

Considering the high spatial variability in the observed changes, no unified approach will work, but specific tailormade interventions are needed for the most affected subcatchments and main catchments. Future investigations should conduct a careful basin-wide assessment of benefits derived from water use, and assess the first priority water uses, including commercial forest plantations, which by default are priority users.

Acknowledgements. The authors would like to thank the UNESCO-IHE Partnership Research Fund (UPaRF), through the RISKOMAN project, for funding of this research. The Water Research Commission of South Africa, through project K5/1935, also contributed additional funding, which is appreciated. All partners of the RISKOMAN project (University of KwaZulu-Natal UKZN, ICMA, Komati Basin Water Authority KOBWA, Eduardo Mondlane University), including the reference group, are thanked for their valuable inputs. Streamflow and rainfall data were kindly provided by DWA, UKZN and SAWS.

Edited by: D. Mazvimavi 


\section{References}

Beumer, J. and Mallory, S.: Water Requirements and Availability Reconciliation Strategy for the Mbombela Municipal Area. Final Reconcialiation Strategy, Department of Water Affairs, South Africa, 2014.

Birkel, C., Soulsby, C., Ali, G., and Tetzlaff, D.: Assessing the cumulative impacts of hydropower regulation on the flow characteristics of a large atlantic Salmon river system, River Res. Applic., 30, 456-475, doi:10.1002/rra.2656, 2014.

Bunn, S. E. and Arthington, A. H.: Basic Principles and Ecological Consequences of Altered Flow Regimes for Aquatic Biodiversity, Environ. Manage., 30, 492-507, doi:10.1007/s00267-0022737-0, 2002.

De Winnaar, G. and Jewitt, G.: Ecohydrological implications of runoff harvesting in the headwaters of the Thukela River basin, South Africa, Phys. Chem. Earth, Parts A/B/C, 35, 634-642, doi:10.1016/j.pce.2010.07.009, 2010.

DWAF - Department of Water Affairs and Forestry: Inkomati Water Availability Assessment Study, Water Requirements Volume 1, prepared by: Water for Africa Environmental, Engineering and Management Consultants, SRK Consulting and CPH20, PWMA 05/X22/00/0908, Pretoria, 2009a.

DWAF - Department of Water Affairs and Forestry: Inkomati Water Availability Assessment Study, Main Report, prepared by: Water for Africa Environmental, Engineering and Management Consultants, SRK Consulting and CPH20, PWMA 05/X22/00/0808, Pretoria, 2009b.

DWAF - Department of Water Affairs and Forestry: Inkomati Water Availability Assessment Study, Hydrology of Sabie River Volume 1, prepared by: Water for Africa Environmental, Engineering and Management Consultants, SRK Consulting and CPH20, PWMA 05/X22/00/1608, Pretoria, 2009c.

DWAF - Department of Water Affairs and Forestry: Inkomati Water Availability Assessment Study, Hydrology of Crocodile River Volume 1, prepared by: Water for Africa Environmental, Engineering and Management Consultants, SRK Consulting and CPH20, PWMA 05/X22/00/1508, Pretoria, 2009d.

Fanta, B., Zaake, B. T., and Kachroo, R. K.: A study of variability of annual river flow of the southern African region, Hydrol. Sci. J., 46, 513-524, doi:10.1080/02626660109492847, 2001.

Guzman, J. A. and Chu, M. L.: SPELL-stat v 15110 B. Grupo en Prediccion y Modelamiento Hidroclimatico Universidad Industrial de Santander, Colombia, Hydrological Modelling and Prediction Group, Industrial University of Santander, Colombia, available at: http://jguzman.info/legacy/index.html, 2003.

$\mathrm{Hu}$, Y., Maskey, S., Uhlenbrook, S., and Zhao, H.: Streamflow trends and climate linkages in the source region of the Yellow River, China, Hydrol. Process., 25, 3399-3411, doi:10.1002/hyp.8069, 2011.

Hughes, D. A. and Mallory, S. J. L.: Including environmental flow requirements as part of real-time water resource management, River Res. Applic., 24, 852-861, doi:10.1002/rra.1101, 2008.

Hughes, D. A., Tshimanga, R. M., Tirivarombo, S., and Tanner, J.: Simulating wetland impacts on stream flow in southern Africa using a monthly hydrological model, Hydrol. Process., 28, 17751786, doi:10.1002/hyp.9725, 2014.

ICMA: The Inkomati Catchment Management Strategy: A First Generations Catchment Management Strategy for the Inkomati
Water Management Area, Inkomati Catchment Management Agency, Nelspruit, 2010.

Jarmain, C., Dost, R. J. J., De Bruijn, E., Ferreira, F., Schaap, O., Bastiaanssen, W. G. M., Bastiaanssen, F., van Haren, I., van Haren, I. J., Wayers, T., Ribeiro, D., Pelgrum, H., Obando, E., and Van Eekelen, M. W.: Spatial Hydro-meteorological data for transparent and equitable water resources management in the Incomati catchment, Pretoria, South Africa, 2013.

Jewitt, G. P. W.: The 8\%-4\% debate: Commercial afforestation and water use in South Africa, S. Afr. Forest. J., 194, 1-5, 2002.

Jewitt, G.: Integrating blue and green water flows for water resources management and planning, Phys. Chem. Earth, Parts A/B/C, 31, 753-762, http://dx.doi.org/10.1016/j.pce.2006.08. 033, 2006a.

Jewitt, G.: Water and Forests, in: Encyclopedia of Hydrological Sciences, John Wiley \& Sons, Ltd, 2006b.

Jewitt, G. P. W., Garratt, J. A., Calder, I. R., and Fuller, L.: Water resources planning and modelling tools for the assessment of land use change in the Luvuvhu Catchment, South Africa, Phys. Chem. Earth, Parts A/B/C, 29, 1233-1241, 2004.

Kruger, A. C. and Shongwe, S.: Temperature trends in South Africa: 1960-2003, Int. J. Climatol., 24, 1929-1945, doi:10.1002/joc.1096, 2004.

LeMarie, M., van der Zaag, P., Menting, G., Baquete, E., and Schotanus, D.: The use of remote sensing for monitoring environmental indicators: The case of the Incomati estuary, Mozambique, Phys. Chem. Earth, Parts A/B/C, 31, 857-863, doi:10.1016/j.pce.2006.08.023, 2006.

Lennard, C., Coop, L., Morison, D., and Grandin, R.: Extreme events: Past and future changes in the attributes of extreme rainfall and the dynamics of their driving processes, Climate Systems Analysis Group University of Cape Town, 2013.

Love, D., Uhlenbrook, S., Twomlow, S., and van Der Zaag, P.: Changing hydroclimatic and discharge patterns in the northern Limpopo Basin, Zimbabwe, Water SA, 36, 335-350, 2010.

Lynch, S. D.: The Development of a Raster Database of Annual, Monthly and Daily Rainfall for Southern Africa., Water Research Commission, South Africa, Rep. 1156/1/04Rep. 1156/1/04, 2003.

Maingi, J. K. and Marsh, S. E.: Quantifying hydrologic impacts following dam construction along the Tana River, Kenya, J. Arid Environ., 50, 53-79, doi:10.1006/jare.2000.0860, 2002.

Masih, I., Uhlenbrook, S., Maskey, S., and Smakhtin, V.: Streamflow trends and climate linkages in the Zagros Mountains, Iran, Clim. Change, 104, 317-338, doi:10.1007/s10584-009-9793-x, 2011.

Mathews, R. and Richter, B. D.: Application of the Indicators of Hydrologic Alteration Software in Environmental Flow Setting1, JAWRA J. Am. Water Resour. Assoc., 43, 1400-1413, doi:10.1111/j.1752-1688.2007.00099.x, 2007.

Miao, C. Y., Shi, W., Chen, X. H., and Yang, L.: Spatiotemporal variability of streamflow in the Yellow River: possible causes and implications, Hydrol. Sci. J., 57, 1355-1367, doi:10.1080/02626667.2012.718077, 2012.

Milly, P. C. D., Betancourt, J., Falkenmark, M., Hirsch, R. M., Kundzewicz, Z. W., Lettenmaier, D. P., and Stouffer, R. J.: Stationarity Is Dead: Whither Water Management?, Science, 319, 573-574, doi:10.1126/science.1151915, 2008. 
Montanari, A., Young, G., Savenije, H. H. G., Hughes, D., Wagener, T., Ren, L. L., Koutsoyiannis, D., Cudennec, C., Toth, E., Grimaldi, S., Blöschl, G., Sivapalan, M., Beven, K., Gupta, H., Hipsey, M., Schaefli, B., Arheimer, B., Boegh, E., Schymanski, S. J., Di Baldassarre, G., Yu, B., Hubert, P., Huang, Y., Schumann, A., Post, D. A., Srinivasan, V., Harman, C., Thompson, S., Rogger, M., Viglione, A., McMillan, H., Characklis, G., Pang, Z., and Belyaev, V.: Panta Rhei - Everything Flows: Change in hydrology and society - The IAHS Scientific Decade 2013-2022, Hydrolog. Sci. J., 58, 1256-1275, doi:10.1080/02626667.2013.809088, 2013.

Moore, D., Dore, J., and Gyawali, D.: The World Commission on Dams + 10: Revisiting the large dam controversy, Water Altern., 3, 3-13, 2010.

Mukororira, F.: Analysis of water allocation in the Komati catchment downstream of Maguga and Driekoppies Dams, MSc Thesis WM 12.18, Water Management, UNESCO-IHE, Delft, 2012.

Mussá, F., Zhou, Y., Maskey, S., Masih, I., and Uhlenbrook, S.: Trend analysis on dry extremes of precipitation and discharge in the Crocodile River catchment, Incomati basin, 14th WaterNet/ WARFSA/GWP-SA symposium, 30 October-1 November 2013, Dar es Salaam, Tanzania, 2013,

Nkomo, S. and van der Zaag, P.: Equitable water allocation in a heavily committed international catchment area: the case of the Komati Catchment, Phys. Chem. Earth, Parts A/B/C, 29, 13091317, 2004.

Pettitt, A.: A non-parametric approach to the change-point problem., Appl. Stat., 28, 126-135, 1979.

Pollard, S. and du Toit, D.: Integrated water resource management in complex systems: How the catchment management strategies seek to achieve sustainability and equity in water resources in South Africa, Water SA, 34, 671-680, 2009.

Pollard, S. and du Toit, D.: Towards the sustainability of freshwater systems in South Africa: An exploration of factors that enable and constrain meeting the ecological Reserve within the context of Integrated Water Resources Management in the catchments of the lowveld, Water Research Comission, Pretoria, South Africa. Report No. TT 477/10, 2011a.

Pollard, S. and du Toit, D.: Towards Adaptive Integrated Water Resources Management in Southern Africa: The Role of Selforganisation and Multi-scale Feedbacks for Learning and Responsiveness in the Letaba and Crocodile Catchments, Water Resour. Manage., 25, 4019-4035, doi:10.1007/s11269-011-9904-0, 2011b.

Pollard, S., Du Toit, D., and Biggs, H.: River management under transformation: The emergence of strategic adaptive management of river systems in the Kruger National Park, Koedoe, 53, 1011, doi:10.4102/koedoe.v53i2.1011, 2011.

Pollard, S., Mallory, S., Riddell, E., and Sawunyama, T.: Towards improving the assessment and implementation of the reserve: real-time assessment and implementation of the ecological reserve: report to the Water Research Commission, Water Research Commission, Pretoria, South Africa, 2012.

Richter, B. D. and Thomas, G. A.: Restoring environmental flows by modifying dam operations, Ecol. Soc., 12, available at: http: //www.ecologyandsociety.org/vol12/iss1/art12/, 2007.

Richter, B. D., Baumgartner, J. V., Powell, J., and Braun, D. P.: A Method for Assessing Hydrologic Alteration within Ecosystems, Conserv. Biol., 10, 1163-1174, 1996.
Richter, B. D., Baumgartner, J. V., Braun, D. P., and Powell, J.: A spatial assessment of hydrologic alteration within a river network, Regulated Rivers: Res. Manage., 14, 329-340, doi:10.1002/(sici)1099-1646(199807/08)14:4< 329::aid-rrr505> 3.0.co;2-e, 1998.

Richter, B. D., Mathews, R., Harrison, D. L., and Wigington, R.: Ecologically sustainable water management: managing river flows for ecological integrity, Ecol. Appl., 13, 206224, doi:10.1890/1051-0761(2003)013[0206:eswmmr]2.0.co; 2, 2003.

Riddell, E., Pollard, S., Mallory, S., and Sawunyama, T.: A methodology for historical assessment of compliance with environmental water allocations: lessons from the Crocodile (East) River, South Africa, Hydrol. Sci. J., 59, 831-843, doi:10.1080/02626667.2013.853123, 2013.

Rockström, J., Falkenmark, M., Karlberg, L., Hoff, H., Rost, S., and Gerten, D.: Future water availability for global food production: The potential of green water for increasing resilience to global change, Water Resour. Res., 45, W00A12, doi:10.1029/2007wr006767, 2009.

Rouault, M., Fauchereau, N., Pohl, B., Penven, P., Richard, Y., Reason, C., Pegram, G., Phillippon, N., Siedler, G., and Murgia, A.: Multidisciplinary analysis of hydroclimatic variability at the catchment scale, University of Cape Town, CRC, Universite de Dijon, UBO, Universite de Bretagne Occidentale, University of Kwazulu Natal, IMF, University of Kiel, LTHE, Universite de Grenoble, 2010.

Schulze, R. E.: Approaches towards practical adaptive management options for selected water-related sectors in South Africa in a context of climate change, Water SA, 37, 621-645, 2011.

Schulze, R.: A 2011 perspective on climate change and the South African water sector, WRC Report TT 518/12, Gezina, South Africa, 2012.

Shongwe, M. E., van Oldenborgh, G. J., van den Hurk, B. J. J. M., de Boer, B., Coelho, C. A. S., and van Aalst, M. K.: Projected Changes in Mean and Extreme Precipitation in Africa under Global Warming. Part I: Southern Africa, J. Climate, 22, 3819-3837, doi:10.1175/2009jcli2317.1, 2009.

Taylor, V., Schulze, R., and Jewitt, G. P. W.: Application of the Indicators of Hydrological Alteration method to the Mkomazi River, KwaZulu-Natal, South Africa, Afr. J. Aquatic Sci., 28, 111, doi:10.2989/16085914.2003.9626593, 2003.

TPTC: Tripartite Permanent Technical Committee (TPTC) between, Moçambique, South Africa, Swaziland, PRIMA: IAAP 3: Consultancy Services for Integrated Water Resources Management. Baseline evaluation and scoping report: Part C. Report No.: IAAP 3: 03C-2010, Prepared for TPTC by Aurecon, Pretoria, 2010.

Tullos, D., Tilt, B., and Liermann, C. R.: Introduction to the special issue: Understanding and linking the biophysical, socioeconomic and geopolitical effects of dams, J. Environ. Manage., 90, Supplement 3, S203-S207, doi:10.1016/j.jenvman.2008.08.018, 2009.

Van den Berg, E., Plarre, C., Van den Berg, H., and Thompson, M.: The South African national land cover 2000, Agricultural Research Council (ARC) and Council for Scientific and Industrial Research (CSIR), Pretoria, Report No. GW/A/2008/86, 2008. 
Van der Zaag, P. and Vaz, A. C.: Sharing the Incomati waters: cooperation and competition in the balance, Water Pol., 5, 349-368, 2003.

van Eekelen, M. W., Bastiaanssen, W. G. M., Jarmain, C., Jackson, B., Ferreira, F., van der Zaag, P., Saraiva Okello, A., Bosch, J., Dye, P., Bastidas-Obando, E., Dost, R. J. J., and Luxemburg, W. M. J.: A novel approach to estimate direct and indirect water withdrawals from satellite measurements: A case study from the Incomati basin, Agr. Ecosyst. Environ., 200, 126-142, doi:10.1016/j.agee.2014.10.023, 2015.

Vörösmarty, C. J., McIntyre, P., Gessner, M. O., Dudgeon, D., Prusevich, A., Green, P., Glidden, S., Bunn, S. E., Sullivan, C. A., and Liermann, C. R.: Global threats to human water security and river biodiversity, Nature, 467, 555-561, 2010.
Warburton, M. L., Schulze, R. E., and Jewitt, G. P. W.: Confirmation of $A C R U$ model results for applications in land use and climate change studies, Hydrol. Earth Syst. Sci., 14, 2399-2414, doi:10.5194/hess-14-2399-2010, 2010.

Warburton, M. L., Schulze, R. E., and Jewitt, G. P. W.: Hydrological impacts of land use change in three diverse South African catchments, J. Hydrol., 414-415, 118-135, doi:10.1016/j.jhydrol.2011.10.028, 2012.

Zhang, X., Zhang, L., Zhao, J., Rustomji, P., and Hairsine, P.: Responses of streamflow to changes in climate and land use/cover in the Loess Plateau, China, Water Resour. Res., 44, W00A07, doi:10.1029/2007wr006711, 2008. 